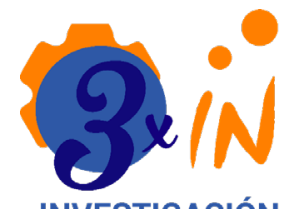

INVESTIGACIÓN E INNOVACION en INGENERIAS ISSN2344-8652

OPEN ACCESS

Recibido: 22/09/2021

Aceptado: 22/10/2021

Publicado: 13/12/2021

Correspondencia de autores: ceromero@unicauca.edu.co

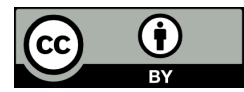

Copyrith 2020 by Investigación e Innovación en Ingenierías

\section{Criterios de ubicación de algoritmos de inteligencia computacional en la web de las cosas: Revisión sistemática de la literatura}

\section{Location criteria for computational intelligence algorithms on the web of things: A systematic review of the literature}

\author{
Camilo Enrique Romero Parra ID Miguel Ángel Niño Zambrano iD \\ Carlos Alberto Cobos Lozada \\ Universidad del Cauca, Colombia
}

Objetivo: Determinar y caracterizar el estado actual del conocimiento acerca de los criterios de ubicación del procesamiento de datos, utilizando técnicas de inteligencia computacional en un Ecosistema de Objetos Inteligentes de la Web de las Cosas. Metodología: La revisión sistemática que se presenta a continuación se basa en los estudios realizados por Petersen y Kitchenham , se plantearon cuatro preguntas de investigación, se aplicó el método PICOC para identificar las palabras clave, se planteó una cadena de búsqueda y cuatro motores de búsqueda, se plantearon los criterios de inclusión y exclusión de estudios primarios, así como los criterios de evaluación de la calidad, la estrategia de extracción de datos y el método de síntesis. Resultados: Se lograron responder las cuatro preguntas de investigación planteadas, encontrando que la mayoría de los estudios carecen de una implementación en las tres ubicaciones analizadas y un único estudio que compara el desempeño obtenido por un algoritmo de inteligencia computacional al procesar información en distintas ubicaciones del ecosistema. Conclusiones: Se demostró la necesidad de continuar realizando estudios en el área de la localización del procesamiento en ecosistemas inteligentes utilizando técnicas de inteligencia computacional para el procesamiento en distintas ubicaciones. Además, se evidencia una necesidad en hacer un mayor énfasis en la comparativa del rendimiento obtenido al realizar implementaciones teniendo en cuenta distintas técnicas de inteligencia computacional.

Palabras clave: Internet de las Cosas, Web de las Cosas, Computación en la niebla, Computación en el borde, Computación en la nube, Inteligencia Computacional.

\section{Abstract}

Objective: Determine and characterize the current state of the knowledge about the location criteria of data processing, using computational intelligence techniques in an Intelligent Objects Ecosystem of Web of Things. Methodology: The systematic review presented below is based on the studies carried out by Petersen and Kitchenham, four research questions were proposed, the PICOC method was applied to identify the keywords, a search chain and four search engines were proposed, the inclusion and exclusion criteria of primary studies were proposed, as well as the quality evaluation criteria, the data extraction strategy and the synthesis method. Results: It was possible to answer the four research questions proposed, finding that most of the studies lack an implementation in the three analyzed locations and a single study compares the performance obtained by a computational intelligence algorithm when processing information in different locations of the ecosystem. Conclusions: The need to continue carrying out studies in the area of the localization of processing in intelligent ecosystems was demonstrated using computational intelligence techniques for processing in different locations. In addition, there is evidence of a need to place greater emphasis on comparing the performance obtained when carrying out implementations taking into account different computational intelligence techniques.

Keywords: Internet of Things (IoT), Web of Things, Fog Computing, Edge Computing, Cloud Computing, Computational Intelligence.

Como citar (IEEE): C. Romero-Parra., M. Niño-Zambrano., y C. Cobos-Lozada. "Criterios de ubicación de algoritmos de inteligencia computacional en la web de las cosas: Revisión sistemática de la literatura”. Investigación e Innovación en Ingenierías, vol. 9, n³, 108-123, 2021. DOI: https://doi.org/10.17081/invinno.9.3.5566 


\section{Introducción}

En años recientes se ha producido un gran interés dentro del área del Internet de las Cosas (Internet of Things - IOT), la cual permite una interacción entre el mundo físico (compuesto por hardware, sensores y actuadores) y el mundo digital (software con capacidad computacional y de conectividad) embebida dentro de un objeto inteligente [1]. Adicionalmente, la Web de las cosas (Web of Things - WoT) permite la integración de objetos inteligentes con servicios que ofrece la Web [2], comunicándose por medio de protocolos bien definidos y permitiendo que un usuario pueda acceder a una gran variedad de servicios y recursos. Esto depende del contexto determinado por las necesidades específicas de cada usuario $[3,4]$.

Actualmente, se está llevando el desarrollo de la loT hacia los ecosistemas de objetos inteligentes de la loT [5]. Estos ecosistemas consisten en un conjunto de objetos con capacidades de razonamiento, procesamiento y comunicación, que se interconectan entre sí para generar servicios interoperables entre ellos. Uno de los principales desafíos para desarrollar este tipo de sistemas es el problema de la heterogeneidad profunda [6].

Dentro de las arquitecturas de la loT destaca la computación en la nube (Cloud Computing) [7], la cual ofrece la posibilidad de acceder a una amplia variedad de servicios a través de Internet, con la ventaja de que los datos provistos por dispositivos y usuarios sean accedidos desde cualquier lugar y estar disponibles sin requerir de una instalación de software previa en un dispositivo, reduciendo los costos que esto conlleva.

Una de las principales falencias encontradas en el estado del arte es la falta de distinción entre las arquitecturas IoT en el borde y en la niebla, siendo términos que los propios autores deciden intercambiar y asumir como iguales $[8,9,10]$. La computación en el borde (Edge Computing) y la computación en la niebla (Fog Computing) son arquitecturas que extienden la arquitectura del Cloud Computing. El Edge Computing puede procesar los datos adquiridos en el punto de origen mediante una aplicación IoT, teniendo las capacidades de procesamiento y comunicación directamente dentro del dispositivo inteligente [11], mientras que en el Fog Computing hay un único dispositivo centralizado responsable del procesamiento, que se encarga de analizar los datos de diferentes puntos finales en la red, tomando la información de los dispositivos inteligentes ubicados en el borde [12].

La ventaja que presenta la computación en el borde frente a la computación en la nube está en los tiempos de respuesta, los cuales pueden reducirse gracias a que el procesamiento se hace de manera local tan pronto como se adquieren los datos por parte del dispositivo inteligente. Por otro lado, la ventaja que presenta la computación en la nube frente a la computación en la niebla está en la capacidad de procesamiento y almacenamiento de datos [13].

Dado lo anterior, los diseñadores de aplicaciones loT tienen inconvenientes al momento de decidir en qué sitio de la red desplegar los diferentes algoritmos de procesamiento de datos. Normalmente toman la decisión de manera empírica, lo cual en muchos casos genera retrasos de desarrollo, o en el peor de los casos, aplicaciones que no cumplen con los requisitos de eficiencia [14, 15].

Por esta razón, sería importante encontrar una herramienta, para que los desarrolladores de aplicaciones IoT puedan contar con un conjunto de criterios estandarizados, que permita orientarlos en la decisión de las ubicaciones óptimas para desplegar los algoritmos de procesamiento de datos dentro de un Ecosistema de Objetos Inteligentes de la WoT (Intelligent Objects Ecosystem of Web of Things - IOEoWoT), de forma que se puedan aprovechar los beneficios propuestos por las tres arquitecturas mencionadas. 
La presente revisión, tiene como objetivo determinar y caracterizar el estado actual del conocimiento acerca de los criterios de ubicación del procesamiento de datos, utilizando técnicas de inteligencia computacional en un IOEOWoT.

A continuación, en la Sección 2 se presenta el protocolo de investigación utilizado para que la revisión sea formal y objetiva. Posteriormente en la Sección 3 se realiza el análisis de la información obtenida mediante tablas comparativas, en la cual se identifican limitaciones, innovación, ubicaciones y demás elementos que sean relevantes para tomar la decisión de que algoritmo implementar y en dónde ubicarlo en la arquitectura IOT. Finalmente, en la Sección 4 se presentan las conclusiones de la revisión.

\section{Metodología}

Las revisiones sistemáticas, también conocidas como estudios de alcance, permiten obtener una visión general de un área de investigación con el fin de establecer la cantidad de evidencia sobre un tema específico. Mediante las revisiones sistemáticas se pueden determinar áreas donde un estudio primario es más apropiado. La revisión sistemática que se presenta a continuación se basa en los estudios realizados por Petersen [16], que se basa a su vez en Kitchenham [17].

En la Subsección 2.1 se describen los pasos que componen la etapa de planificación. En la Subsección 2.2 se describen los pasos que componen la etapa de ejecución.

\section{Planificación}

Dentro de la etapa de planificación se describen todos los pasos realizados para llevar a cabo la búsqueda y el análisis de los estudios tomados en cuenta para esta revisión sistemática. En la Subsección 2.1.1 se identifican las preguntas de investigación que se intentan responder por medio de la revisión sistemática. En la Subsección 2.1.2 se aplica el método PICOC para identificar las palabras clave que se utilizarán en la cadena de búsqueda. En la Subsección 2.1.3 se especifican los motores de búsqueda utilizados, junto con la cadena de búsqueda y se muestra el número de resultados obtenidos mediante la búsqueda.

\section{Preguntas de Investigación}

El objetivo de la revisión sistemática es determinar ¿cuáles son los criterios que se deben tener en cuenta a la hora de determinar la ubicación (Edge, Fog, o Cloud) de los algoritmos de inteligencia computacional en un IOEoWoT para realizar el procesamiento de datos? Con esto en mente, se plantean las siguientes preguntas de investigación (Research Questions RQs):

- RQ1: ¿Qué técnicas de inteligencia computacional son utilizadas para realizar la toma y procesamiento de datos dentro de los ecosistemas inteligentes que se mencionan en cada estudio?

- - RQ2: ¿Qué mejora presentan los criterios frente a las técnicas preexistentes en la literatura?

- RQ3: ¿Cuáles son los criterios de localización del procesamiento de datos utilizando técnicas de inteligencia computacional?

- - RQ4: ¿Cuáles son las principales limitaciones que presentan los estudios a la hora de localizar el procesamiento de datos dentro de un IOEoWoT? 
Una vez realizado el análisis, se busca identificar las carencias existentes en el área para el planteamiento de estudios futuros.

\section{Método PICOC}

El modelo PICOC (Población Intervención Comparación Salidas y Contexto) sugerido por Kitchenham [17], fue desarrollado para identificar las palabras clave y formular la cadena de búsqueda a partir de las preguntas de investigación.

- Población: En el caso de la localización del procesamiento de datos en un ecosistema inteligente, se refiere a estudios que, mediante el uso de técnicas de inteligencia computacional buscan dar solución a un escenario de aplicación. En el caso de la presente revisión sistemática, se buscan estudios que realicen el análisis de los datos priorizando las capacidades de procesamiento de los recursos hardware y software presentes en el ecosistema.

- Intervención: Se refiere a la aplicación de técnicas de inteligencia computacional y el desempeño obtenido al realizar dicho procesamiento en diferentes ubicaciones del ecosistema (diferentes dispositivos hardware y software). En el caso del presente estudio, se busca determinar los criterios que permitan localizar un procesamiento especifico.

- Comparación: En este estudio se comparan los criterios aplicados para ubicar el procesamiento dentro del ecosistema y las debilidades que se pueden identificar. Dentro de los criterios que proponga cada estudio, se espera encontrar una comparativa del desempeño alcanzado por la técnica utilizada en las diferentes ubicaciones (Cloud, Fog o Edge).

- Salidas: No se estipulan salidas como objetivo de esta revisión sistemática. El objetivo del presente estudio es realizar una revisión de la literatura existente referente al tópico de interés, y clasificar las soluciones existentes.

- Contexto: Se busca identificar qué criterios se han propuesto para mitigar el problema que se presenta a la hora de querer ubicar el procesamiento de los datos cuando se implementan técnicas de inteligencia computacional soportadas en servicios alojados en la nube, en el Fog o en el borde del ecosistema.

- Las palabras clave identificadas son: Web de las cosas (WoT), internet de las cosas (IoT), Fog Computing, Edge Computing, inteligencia computacional, Offloading (y sus sinónimos: localización y ubicación).

\section{Estrategia de Búsqueda}

Con base en las preguntas de investigación planteadas y las palabras clave identificadas a partir del método PICOC, se estipula la siguiente cadena de búsqueda, la cual fue utilizada en los motores de búsqueda: ScienceDirect, Google Schoolar y Springer.

("Fog computing") AND ("Edge computing") AND ("Internet of Things") AND ("Context awareness") AND ("Artificial Intelligence") AND ("Offloading" OR "Location" OR "Placement")

Cabe resaltar que los estudios que incluyen arquitecturas de Fog Computing y Edge Computing derivan, como se dijo anteriormente, del Cloud Computing, razón por la cual no es necesaria su integración en la cadena de búsqueda. 
Adicionalmente, esta cadena de búsqueda se adaptó para el motor de búsqueda IEEE de la siguiente manera: ("All Metadata": Cloud computing) AND ("All Metadata": Internet of Things) AND ("All Metadata": Context awareness) AND ("All Metadata": Artificial Intelligence)

Con el fin de llevar un registro ordenado de las referencias utilizadas en la presente revisión, se utilizó el gestor de referencias Mendeley. Éste estudio se ha realizado durante el último trimestre de 2020 y el primer trimestre de 2021. En la Tabla 1 se puede apreciar el número de resultados obtenidos al aplicar la cadena de búsqueda en las bases de datos seleccionadas.

Tabla 1: Número de estudios por base de datos

\begin{tabular}{|c|c|}
\hline Bases de datos & Resultados de búsqueda \\
\hline Google Scholar & 406 \\
\hline IEEE & 13 \\
\hline ScienceDirect & 173 \\
\hline Springer Link & 2 \\
\hline
\end{tabular}

Fuente: Autoría propia

\section{Criterios de Selección de Estudios Primarios}

Los estudios fueron seleccionados a partir de su título y resumen, así como, la lectura de texto completo y la evaluación de calidad (rigurosidad, metodología de investigación implementada, objetividad en el análisis de resultados y limitaciones tenidas en cuenta). La revisión sistemática fue realizada por un único autor, siendo esta una limitación asociada a la confiabilidad de la revisión. Previo a la primera revisión de literatura se identificaron artículos tipo revisión de literatura (Surveys), asociados con las palabras clave identificadas, con el fin de tener claridad frente al tema a tratar y hacia el objetivo propuesto. Frente a dudas que se presentaron conforme se llevaba a cabo la primera revisión de la literatura, se procedió a realizar una lectura más rigurosa, mejorando la calidad de la exclusión de estudios. Se estipulo una ventana de tiempo de 5 años (desde el año 2016 hasta el 2021), para realizar la búsqueda de estudios, dado que la temática a tratar se encuentra en constante evolución y es un campo muy reciente dentro del contexto de ecosistemas de objetos inteligentes de la WoT.

Los criterios de inclusión que se tomaron en cuenta son los siguientes:

- Los estudios están dentro del campo de la localización del procesamiento de datos utilizando técnicas de inteligencia computacional en ecosistemas inteligentes con conocimiento del contexto.

- Los estudios proponen criterios claros para realizar la localización del procesamiento de datos.

- Los estudios se basan en ecosistemas inteligentes que consumen servicios alojados en la nube y sus derivados.

- Los estudios hacen uso de dispositivos inteligentes para realizar la toma de datos.

- Los criterios de exclusión que se tomaron en cuenta son los siguientes:

- El estudio no se encuentra en idioma inglés.

- El estudio no pertenece a la categoría de articulo científico.

- El estudio se encuentra duplicado o desactualizado.

- El estudio no trata el tema de interés. 


\section{Criterios de Evaluación de Calidad}

Con el fin de evaluar la calidad de los estudios incluidos se plantearon las siguientes preguntas, con su correspondiente rango de valoración:

- ¿Los criterios de localización propuestos fueron puestos a prueba en un caso de estudio?

$\gg \mathrm{Si}-5.0$

$\Rightarrow$ No -0.0

- ¿Los criterios de localización propuestos se basa en una comparativa clara de un mismo procesamiento realizado en distintas ubicaciones de un mismo ecosistema?

$\gg$ Efectivamente - Entre 4.0 y 5.0

$\gg$ Parcialmente - Entre 2.0 y 4.0

$\gg$ No se realiza comparativa 0.0

- Criterio de citación: Para artículos publicados durante 2021 no se tiene en cuenta el criterio de evaluación.

Para artículos publicados entre 2018 y 2020:

$\gg$ De 10 a más citaciones - Entre 4.0 y 5.0

$\gg$ Entre 5 y 10 citaciones - Entre 2.0 y 4.0

$\gg$ Entre 0 y 5 citaciones - Entre 0.0 y 2.0

Para artículos publicados entre 2015 y 2018:

$\gg$ De 20 a más citaciones - Entre 4.0 y 5.0

$\Rightarrow$ Entre 10 y 20 citaciones - Entre 2.0 y 4.0

$\gg$ Entre 0 y 10 citaciones - Entre 0.0 y 2.0

¿Se realiza una comparación clara de los resultados obtenidos al aplicar métodos propuestos por otros autores?

$\Longrightarrow$ De manera clara - Entre 2.5 y 5.0

$\gg$ De manera ambigua - Entre 0.0 y 2.5

\section{Estrategia de Extracción de Datos}

Con el fin de categorizar como los estudios solucionan las preguntas de investigación planteadas, se buscará agrupar la manera en que los estudios den su repuesta a cada pregunta. En caso de no presentarse una generalidad, se realizará el análisis de las respuestas dadas y se destacarán las principales aportaciones realizadas. 


\section{Métodos de Sintesis}

Los resultados obtenidos al categorizar los estudios primarios seleccionados se muestran en la Sección 3 mediante la respuesta especifica que plantea cada estudio a las preguntas de investigación propuestas.

\section{Ejecución}

Cómo se estipuló en la sección anterior, los pasos que se llevaron a cabo durante la revisión consistieron en:

1. Aplicar la cadena de búsqueda definida en la Subsección 2.1.3 en los motores de búsqueda seleccionados.

2. Aplicar los criterios de inclusión y exclusión estipulados en la Subsección 2.1.4 sobre los resultados obtenidos, a partir del resumen, título y conclusiones de los estudios, pero sin limitarse a estas tres secciones del documento cuándo se requería un mayor entendimiento del mismo. De esta manera se pasó de tener 594 estudios a tener $48[8,9,10,14,15,18,19,20,21,22,23,24,25,26,27,28,29,30,31,32,33,34]$. La principal razón por la cual se realizó la exclusión de estos artículos fue la falta de claridad a la hora de proponer criterios para realizar la localización del procesamiento de datos, al priorizar en objetivos diferentes como pueden ser, por ejemplo, la disminución en el tiempo de respuesta [35, 36, 37, 38, 39, 40, 41, $42,43,44,45,46,47,48,49,50,51,52,53,54,55,56,57,58,59,60,61]$, o la seguridad de los datos [62].

3. Tras leer los documentos por completo, y habiendo aplicado nuevamente los criterios de inclusión y exclusión, se identificaron 16 estudios primarios $[9,46,47,48,49,50,51,52,53,54,55,56,57,58,59,60]$, descartando 32 estudios que no cumplían con los criterios de inclusión y exclusión.

4. Finalmente, se realizó la evaluación de la calidad de cada uno de los estudios primarios y su caracterización dependiendo de la respuesta a la que llegaron respecto a las preguntas de investigación planteadas.

\section{Resultados}

\section{Técnicas de inteligencia computacional utilizadas para el procesamiento de datos}

En la Figura 1, se muestra de manera gráfica el número de estudios que tienen en cuenta el Cloud, Fog y/o Edge Computing como una posible ubicación para el procesamiento. De igual forma se muestra el número de estudios que utilizan técnicas de inteligencia computacional para realizar el procesamiento de los datos dentro del ecosistema inteligente (Procesamiento con Inteligencia Computacional - PIC), el número de estudios que comparan el rendimiento de diferentes técnicas de inteligencia computacional (Comparativa de Inteligencia Computacional - CIC), y el número de estudios que cuentan con una implementación de los criterios propuestos mediante una aplicación loT. 
Figura 1. Ubicación y procesamiento de datos

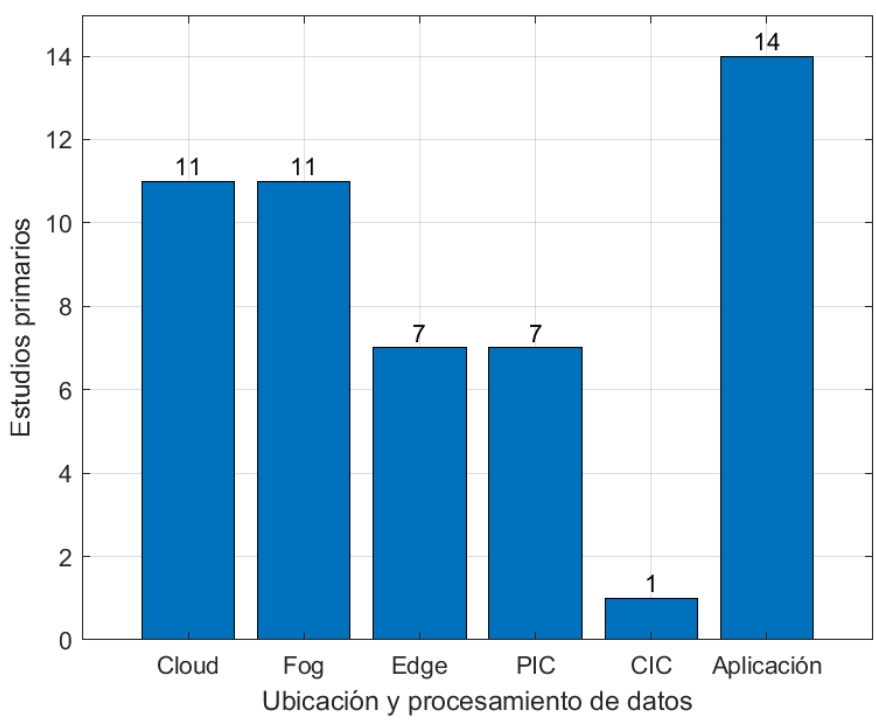

Fuente: Autoría propia

Como se puede apreciar, un único estudio primario realiza la comparativa de distintas técnicas de Inteligencia Computacional (IC) [9] y siete de ellos utilizan dichas técnicas para realizar el procesamiento de datos en soluciones particulares [9, 20, 21, 22, 23, 24, 25].

Adicional a los 7 artículos que realizan procesamiento de datos con técnicas de IC, se presentan 3 artículos que, si bien no presentan una aplicación del método propuesto, hacen referencia a la posibilidad de utilizar estas técnicas dependiendo del escenario de aplicación [14, 26, 27].

Dentro de las técnicas de inteligencia computacional utilizadas predominan el Machine Learning (ML) y el Deep Learning (DL). Además, algunos estudios utilizan metaheurísticas, sin embargo, se evidencia una tendencia al uso de ML y DL.

Dentro de los artículos estudiados se puede apreciar una tendencia al uso de procesamiento distribuido, el cual tiene como objetivo repartir la carga computacional entre los dispositivos que conforman la arquitectura, aprovechando las capacidades de procesamiento de las distintas ubicaciones. Sin embargo, el procesamiento individual que pueda realizar cada dispositivo se deja de lado frecuentemente, siendo esto una limitación, dado que no en todos los casos resulta eficiente repartir la carga computacional.

\section{Innovación y criterios presentes en la literatura}

Respondiendo la pregunta de investigación (RQ2), dentro de los estudios primarios estudiados se encontró, que los criterios de ubicación del procesamiento se basan en tres (3) aspectos fundamentales: Las características del nodo, las tareas asignadas, y las características de la red. Los dispositivos inteligentes tendrán características hardware relacionadas con la capacidad de procesamiento, la capacidad de almacenamiento y la capacidad de interactuar con sus semejantes estableciendo una comunicación colaborativa. Las tareas variarán en el tiempo requerido para ser desarrolladas y en las características propias de cada tarea, como puede ser su complejidad y los requerimientos del dispositivo para cumplirla. Se 
observa que, algunos estudios categorizan las tareas con el fin de asignar un determinado número de tareas a los dispositivos en el Edge, a los nodos Fog, y al Cloud. Otros estudios proponen algoritmos enfocados a la distribución de las tareas de manera automática. Las características de la red se fundamentan en su elasticidad, refiriéndose este término a la escalabilidad de la red, y a su eficiencia, la cual puede variar dependiendo de la infraestructura sobre la cual se soporte el servicio.

Respondiendo la pregunta de investigación (RQ3), la mayor innovación que presentan los estudios primarios se basa en la optimización del procesamiento dentro de las arquitecturas implementadas mediante la descentralización del procesamiento, utilizando el Cloud, el Fog y el Edge Computing, de manera que se aproveche el potencial de procesamiento disponible dentro del ecosistema. De esta manera los estudios logran obtener mejores tiempos de respuesta al solicitar un servicio, mejoras en el consumo energético y mayor seguridad.

Los resultados obtenidos demuestran la necesidad de continuar realizando estudios en el área de la localización del procesamiento en ecosistemas inteligentes. Además, se evidencia una necesidad en hacer un mayor énfasis en la comparativa del rendimiento obtenido en distintas ubicaciones.

\section{Limitaciones}

En la Tabla2se muestran las principales limitaciones presentes en cada estudio, referentes al posicionamiento del procesamiento dentro de cada ecosistema inteligente, las técnicas de inteligencia computacional (IC) mencionadas dentro del estudio, categorizadas como Machine Learning (ML), Deep Learning (DL), Federated Learning (FL), Metaheuristics (MH), y Expert System (ES); la comparación del desempeño obtenido por distintas técnicas de inteligencia computacional implementadas en distintas ubicaciones del ecosistema inteligente (CDU), así como limitaciones propias de cada estudio, dando respuesta a la pregunta de investigación (RQ4).

Tabla 2. Limitaciones

\begin{tabular}{|c|c|c|c|c|c|c|c|c|c|c|}
\hline \multirow{2}{*}{ Estudio } & \multicolumn{3}{|c|}{ Ubicaciones estudiadas } & \multicolumn{5}{|c|}{ Técnicas de IC } & \multirow{2}{*}{ CDU } & \multirow{2}{*}{ Otras limitaciones } \\
\hline & Cloud & Fog & Edge & ML & DL & FL & MH & ES & & \\
\hline [47] & & $x$ & & $x$ & & & & & & $\begin{array}{l}\text { No se tiene en cuenta la comunicación con la } \\
\text { nube }\end{array}$ \\
\hline [13] & $x$ & $x$ & $\mathrm{x}$ & & & & & & & \\
\hline [49] & & $x$ & & $x$ & $x$ & & & & & $\begin{array}{l}\text { No se especifica en que consiste la tarea } \\
\text { asignada }\end{array}$ \\
\hline [50] & $x$ & $x$ & & & & & & & & $\begin{array}{l}\text { No se especifica en que consiste la tarea } \\
\text { asignada } \\
\text { No se toman en cuenta las características } \\
\text { hardware }\end{array}$ \\
\hline [51] & $x$ & & $x$ & & & & & & & \\
\hline [60] & $\mathrm{x}$ & $x$ & & & & & & & & $\begin{array}{l}\text { Se plantea la posibilidad de usar machine } \\
\text { Learning mas no se implementa }\end{array}$ \\
\hline [46] & & & $x$ & $\mathrm{x}$ & & & $x$ & & & $\begin{array}{l}\text { Las tareas que cumplen los dispositivos se } \\
\text { limitan a comunicar información y a él envió } \\
\text { de ordenes }\end{array}$ \\
\hline
\end{tabular}




\begin{tabular}{|c|c|c|c|c|c|c|c|c|}
\hline [52] & $x$ & & & & & $x$ & & \\
\hline [53] & $x$ & & $x$ & & & & & $\begin{array}{l}\text { Los conceptos de Edge y Fog no se diferencian } \\
\text { claramente } \\
\text { No reduce la carga de transporte de datos }\end{array}$ \\
\hline [9] & $x$ & $x$ & & $x$ & $x$ & & $x$ & \\
\hline [54] & & & $x$ & $x$ & $x$ & & & $\begin{array}{l}\text { Los conceptos de Edge y Fog no se diferencian } \\
\text { claramente }\end{array}$ \\
\hline [55] & & $x$ & $x$ & $x$ & & & & $\begin{array}{l}\text { Los conceptos de Edge y Fog no se diferencian } \\
\text { claramente } \\
\text { No se realiza una clasificación exhaustiva del } \\
\text { hardware necesario }\end{array}$ \\
\hline [56] & $x$ & $x$ & & & & & & $\begin{array}{l}\text { No tiene claridad en el concepto de Fog, } \\
\text { diferenciándolo con servidores MEC }\end{array}$ \\
\hline [57] & $x$ & $x$ & & & & & & \\
\hline [58] & $x$ & $x$ & & $x$ & & $x$ & & No tiene claridad en el concepto de Edge \\
\hline [14] & $x$ & $x$ & $x$ & $x$ & & & & \\
\hline
\end{tabular}

\section{Fuente: Autoría propia}

Como se puede apreciar en la Tabla 2, la mayoría de los estudios se enfocan en una computación apoyada en servicios alojados en la nube y en el Fog, mientras que menos de la mitad de los estudios se enfocan en llevar el procesamiento hacia el Edge. Los estudios no tienen un enfoque comparativo entre el desempeño obtenido al realizar el procesamiento de datos entre distintas ubicaciones del ecosistema inteligente, en su lugar buscan la innovación a través de una mejora en los tiempos de respuesta, un menor consumo energético, entre otras. Estos objetivos se logran al migrar el procesamiento a la nube y al Edge principalmente, utilizando en ocasiones los nodos Fog como concentradores de la información adquirida por los dispositivos inteligentes que forman parte de la arquitectura loT y derivando las tareas verticalmente dentro de la arquitectura.

Otra limitación presente en la literatura está asociada con la seguridad, más específicamente en la falta de verificación de la confiabilidad de los dispositivos inteligentes que interactúan dentro del ecosistema aportando información al borde del despliegue.

Se evidencia una tendencia al uso de Machine Learning y Deep Learning, dejando de lado otras técnicas de Inteligencia Computacional, demostrando un sesgo por parte de la comunidad científica frente a las técnicas que pueden solucionar diferentes necesidades particulares de las aplicaciones estudiadas. Se puede apreciar una oportunidad de investigación al tener en cuenta diferentes técnicas de IC, además de ML y DL, tales como: aprendizaje federado, sistemas expertos y lógica difusa, que permitan lograr un mejor desempeño en el contexto de la ubicación del procesamiento de datos en un ecosistema inteligente.

Dentro de los estudios principales seleccionados se identificó un único estudio que realiza una comparativa entre el desempeño obtenido al realizar el procesamiento en las tres ubicaciones propuestas, sin embargo, el estudio carece de una comparación clara entre distintas técnicas de inteligencia computacional alojadas en cada ubicación, en su lugar utilizan un único algoritmo de inteligencia computacional basado en Machine Learning y se comparan los porcentajes de mejora en los tiempos de respuesta entre una y otra ubicación. 
Una de las mayores limitaciones que presentan los estudios es la falta de claridad en la tarea que se va a desempeñar y del algoritmo de inteligencia computacional que se va a implementar para realizar el procesamiento de los datos. Esta limitación nace a raíz de un enfoque de investigación ajeno a la implementación de técnicas de inteligencia computacional en IOEoWoT.

Al realizar la revisión de la literatura gris arrojada en los distintos motores de búsqueda se identifica una dificultad a la hora de diferenciar el Edge Computing y el Fog Computing, resultando en diferentes definiciones que dificultan la correcta identificación de los estudios primarios. Uno de los casos particulares del uso inadecuado del Edge Computing es el término Mobile Edge Computing (MEC), el cual introduce nodos Edge como concentradores de información en vez de utilizar el Fog Computing.

\section{Evaluación de la calidad}

En la Tabla 3 se muestran los resultados obtenidos por cada estudio al aplicar la evaluación de la calidad mencionada en la subsección 2.1.5. cómo se puede observar la comparativa entre distintas ubicaciones del procesamiento es aplicada únicamente por el artículo [9]. la mayoría de los estudios implementan uno o más casos de estudio sobre el modelo propuesto. La mayoría de los estudios carecen de un gran número de citaciones. finalmente se puede concluir que la mayor calificación coincide con el artículo [9], siendo este identificado como 1 de los principales referentes a tener en cuenta a la hora de realizar la ubicación del procesamiento de algoritmos de inteligencia computacional en ecosistemas inteligentes.

Tabla 3. Evaluación de la calidad

\begin{tabular}{|c|c|c|c|c|}
\hline Estudio & Caso de estudio & Comparación & Citaciones & Calificación \\
\hline$[47]$ & 5 & 0 & 0 & 1,67 \\
\hline$[13]$ & 5 & 0 & 0 & 1,67 \\
\hline$[49]$ & 5 & 0 & 2,5 & 2,5 \\
\hline$[50]$ & 5 & 0 & 2 & 2,33 \\
\hline$[51]$ & 5 & 0 & 0 & 1,67 \\
\hline$[60]$ & 5 & 0 & 5 & 3,33 \\
\hline$[46]$ & 5 & 0 & 0 & 1,67 \\
\hline$[52]$ & 5 & 0 & 1,5 & 2,17 \\
\hline$[53]$ & 0 & 0 & 0,5 & 0,17 \\
\hline$[9]$ & 5 & 5 & 1 & 3,67 \\
\hline$[54]$ & 5 & 0 & 5 & 3,33 \\
\hline$[55]$ & 5 & 0 & 0 & 1,67 \\
\hline$[56]$ & 5 & 0 & 0 & 1,67 \\
\hline$[57]$ & 0 & 0 & 5 & 1,67 \\
\hline$[58]$ & 5 & 0 & 5 & 3,33 \\
\hline$[14]$ & 5 & 0 & 1 & 2 \\
\hline
\end{tabular}

Fuente: Autoría propia 
Criterios de ubicación de algoritmos de inteligencia computacional en la web de las cosas: Revisión

sistemática de la literatura

\section{Conclusiones}

En el presente estudio, se pudo determinar y caracterizar el estado actual del conocimiento acerca de los criterios de ubicación del procesamiento de datos utilizando técnicas de inteligencia computacional en un IOEOWOT. Se presentó un protocolo de investigación detallado para realizar la revisión del estado del arte de manera objetiva. Aplicando los criterios de inclusión y exclusión estipulados en la Subsección 2.1.4 sobre los resultados obtenidos, a partir del resumen, título y conclusiones de los estudios, pero sin limitarse a estas tres secciones del documento cuándo se requería un mayor entendimiento del estudio, se pasó de tener 594 estudios a tener 16 estudios primarios, los cuales fueron revisados por completo.

Dentro de los resultados obtenidos se identificó una tendencia entre los estudios de implementar Machine Learning y el Deep Learning como técnicas de IC, siendo algunos de los algoritmos utilizados: Aprendizaje colaborativo, adaptativo y federado, filtrado de datos, clasificación, analítico, vecinos K-más cercanos, entre otros, dejando de lado otras áreas importantes de la IC cómo los Sistemas expertos, lógica difusa y metaheurísticas. Se puede observar una tendencia entre los estudios principales de buscar disminuir la carga computacional centralizada del procesamiento de datos utilizando tanto el Cloud como el Edge y Fog, sin embargo, se evidencia que pocos estudios implementan las tres ubicaciones y solamente uno de ellos compara el procesamiento realizado entre ellas [9].

Se puede apreciar una oportunidad de investigación al tener en cuenta diferentes técnicas de IC, además de ML y DL, que permitan lograr un mejor desempeño en el contexto especifico de la ubicación del procesamiento de datos en un ecosistema inteligente.

Los resultados obtenidos demuestran la necesidad de continuar realizando estudios en el área de la localización del procesamiento en ecosistemas inteligentes utilizando técnicas de inteligencia computacional para el procesamiento en distintas ubicaciones. Además, se evidencia una necesidad en hacer un mayor énfasis en la comparativa del rendimiento obtenido al realizar implementaciones teniendo en cuenta distintas técnicas de inteligencia computacional.

\section{Referencias bibliográficas}

1. P. Sethi and S. R. Sarangi, "Internet of Things: Architectures, Protocols, and Applications," J. Electr. Comput. Eng., vol. 2017, p. 9324035, 2017, doi: 10.1155/2017/9324035.

2. G. Bai, L. Yan, L. Gu, Y. Guo, and X. Chen, "Context-aware usage control for web of things," Secur. Commun. Networks, vol. 7, no. 12, pp. 2696-2712, 2014, doi: 10.1002/sec.424.

3. A. Wagner, J. L. V. Barbosa, and D. N. F. Barbosa, "A model for profile management applied to ubiquitous learning environments," Expert Syst. Appl., vol. 41, no. 4 PART 2, pp. 2023-2034, Mar. 2014, doi: 10.1016/j. eswa.2013.08.098.

4. L. Yao, "A Propagation Model for Integrating Web of Things and Social Networks," in Service-Oriented Computing - ICSOC 2011 Workshops, 2012, pp. 233-238.

5. P. Pedamkar, "IoT Ecosystem," 2020. https://www.educba.com/iot-ecosystem/ (accessed May 26, 2021).

6. M. Niño Zambrano, "Interacción Semántica de Objetos en la Web de las Cosas," 2013.

7. B. Varghese and R. Buyya, "Next generation cloud computing: New trends and research directions," Futur. Gener. Comput. Syst., vol. 79, pp. 849-861, Feb. 2018, doi: 10.1016/j.future.2017.09.020. 
8. A. Jaddoa, G. Sakellari, E. Panaousis, G. Loukas, and P. G. Sarigiannidis, "Dynamic decision support for resource offloading in heterogeneous Internet of Things environments," Simul. Model. Pract. Theory, vol. 101, p. 102019, May 2020, doi: 10.1016/J.SIMPAT.2019.102019.

9. B.Alturki,S. Reiff-Marganiec, C.Perera, and S. De, "Exploring the Effectiveness of Service Decomposition in Fog Computing Architecture for the Internet of Things," IEEE Trans. Sustain. Comput., p. 1, 2019, doi: 10.1109/TSUSC.2019.2907405.

10. G. M. S. Rahman, M. Peng, S. Yan, and T. Dang, "Learning Based Joint Cache and Power Allocation in Fog Radio Access Networks," IEEE Trans. Veh. Technol., vol. 69, no. 4, pp. 4401-4411, Apr. 2020, doi: 10.1109/ TVT.2020.2975849.

11. N. Abbas, Y. Zhang, A. Taherkordi, and T. Skeie, "Mobile Edge Computing: A Survey," IEEE Internet of Things Journal, vol. 5, no. 1. Institute of Electrical and Electronics Engineers Inc., pp. 450-465, Feb. 2018, doi: 10.1109/JIOT.2017.2750180.

12. Z. Mahmood and M. Ramachandran, "Fog Computing: Concepts, Principles and Related Paradigms," in Fog Computing: Concepts, Frameworks and Technologies, Z. Mahmood, Ed. Cham: Springer International Publishing, 2018, pp. 3-21.

13. C. Mechalikh, H. Taktak, and F. Moussa, "A scalable and adaptive tasks orchestration platform for loT," in 2019 15th International Wireless Communications and Mobile Computing Conference, IWCMC 2019, Jun. 2019, pp. 1557-1563, doi: 10.1109/IWCMC.2019.8766744.

14. B. Rababah, T. Alam, and R. Eskicioglu, "The Next Generation Internet of Things Architecture Towards Distributed Intelligence: Reviews, Applications, and Research Challenges," SSRN Electron. J., Jul. 2020, doi: 10.2139/ssrn.3640136.

15. M. Aazam, S. Zeadally, and K. A. Harras, "Offloading in fog computing for loT: Review, enabling technologies, and research opportunities," Futur. Gener. Comput. Syst., vol. 87, pp. 278-289, Oct. 2018, doi: 10.1016/J.FUTURE.2018.04.057.

16. K. Petersen, S. Vakkalanka, and L. Kuzniarz, "Guidelines for conducting systematic mapping studies in software engineering: An update," in Information and Software Technology, 2015, vol. 64, pp. 1-18, doi: 10.1016/j.infsof.2015.03.007.

17. K. BA and S. Charters, "Guidelines for performing Systematic Literature Reviews in Software Engineering," vol. 2, 2007.

18. Y. Meng, M. Naeem, A. Almagrabi, R. Ali, and H. S. Kim, "Advancing the State of the Fog Computing to Enable 5G Network Technologies," Sensors, vol. 20, p. 1754, 2020, doi: 10.3390/s20061754.

19. S. E. Bibri, "A foundational framework for smart sustainable city development: Theoretical, disciplinary, and discursive dimensions and their synergies," Sustain. Cities Soc., vol. 38, pp. 758-794, Apr. 2018, doi: 10.1016/J.SCS.2017.12.032.

20. P. Fountas and K. Kolomvatsos, "Ensemble based Data Imputation at the Edge," in 2020 IEEE 32nd International Conference on Tools with Artificial Intelligence (ICTAI), 2020, pp. 961-968, doi: 10.1109/ ICTAI50040.2020.00150.

21. T. S. J. Darwish and K. Abu Bakar, "Fog Based Intelligent Transportation Big Data Analytics in The Internet of Vehicles Environment: Motivations, Architecture, Challenges, and Critical Issues," IEEE Access, vol. 6, pp. 15679-15701, Mar. 2018, doi: 10.1109/ACCESS.2018.2815989.

22. J. Schmitt, J. Bönig, T. Borggräfe, G. Beitinger, and J. Deuse, "Predictive model-based quality inspection using Machine Learning and Edge Cloud Computing," Adv. Eng. Informatics, vol. 45, p. 101101, Aug. 2020, doi: 10.1016/J.AEl.2020.101101. 
23. P. Porambage, J. Okwuibe, M. Liyanage, M. Ylianttila, and T. Taleb, "Survey on Multi-Access Edge Computing for Internet of Things Realization," IEEE Commun. Surv. Tutorials, vol. 20, no. 4, pp. 29612991, Oct. 2018, doi: 10.1109/COMST.2018.2849509.

24. M. Tahir, Q. Mamoon Ashraf, and M. Dabbagh, "Towards Enabling Autonomic Computing in IoT Ecosystem," in 2019 IEEE Intl Conf on Dependable, Autonomic and Secure Computing, Intl Conf on Pervasive Intelligence and Computing, Intl Conf on Cloud and Big Data Computing, Intl Conf on Cyber Science and Technology Congress (DASC/PiCom/CBDCom/CyberSciTech), 2019, pp. 646-651, doi: 10.1109/DASC/PiCom/CBDCom/CyberSciTech.2019.00122.

25. J. Mocnej, W. K. G. Seah, A. Pekar, and I.Zolotova, "Decentralised IoT Architecture for Efficient Resources Utilisation," IFAC-PapersOnLine, vol. 51, no. 6, pp. 168-173, Jan. 2018, doi: 10.1016/J.IFACOL.2018.07.148.

26. J. Wang, J. Pan, F. Esposito, P. Calyam, Z. Yang, and P. Mohapatra, "Edge cloud offloading algorithms: Issues, methods, and perspectives," ACM Comput. Surv., vol. 52, no. 1, p. 23, Feb. 2019, doi: $10.1145 / 3284387$.

27. H. Liao et al., "Learning-Based Context-Aware Resource Allocation for Edge-Computing-Empowered Industrial IoT," IEEE Internet ThingsJ., vol.7, no. 5, pp. 4260-4277, May 2020, doi:10.1109/JIOT.2019.2963371.

28. M. Silva et al., "Neighborhood-aware Mobile Hub: An Edge Gateway with Leader Election Mechanism for Internet of Mobile Things," Mob. Networks Appl. 2020, pp. 1-14, Sep. 2020, doi: 10.1007/S11036-02001630-3.

29. J. Zhang, M. Ma, W. He, and P. Wang, "On-demand deployment for loT applications," J. Syst. Archit., vol. 111, p. 101794, Dec. 2020, doi: 10.1016/J.SYSARC.2020.101794.

30. D. Amiri et al., "Optimizing Energy Efficiency of Wearable Sensors Using Fog-assisted Control," Fog Comput. Theory Pract., pp. 245-268, Apr. 2020, doi: 10.1002/9781119551713.CH9.

31. J. I. Benedetto, L. A. González, P. Sanabria, A. Neyem, and J. Navón, “Towards a practical framework for code offloading in the Internet of Things," Futur. Gener. Comput. Syst., vol. 92, pp. 424-437, Mar. 2019, doi: 10.1016/J.FUTURE.2018.09.056.

32. J. Zhang, P. Liu, F. Zhang, H. Iwabuchi, A. A. D. H. E. A. De Moura, and V. H. C. De Albuquerque, "Ensemble Meteorological Cloud Classification Meets Internet of Dependable and Controllable Things," IEEE Internet Things J., vol. 8, no. 5, pp. 3323-3330, Mar. 2021, doi: 10.1109/JIOT.2020.3043289.

33. W. Sun, J. Liu, and Y. Yue, "Al-enhanced offloading in edge computing: When machine learning meets industrial IOT," IEEE Netw., vol. 33, no. 5, pp. 68-74, Sep. 2019, doi: 10.1109/MNET.001.1800510.

34. A. S. Gowri and P. Shanth I Bala, "An agent based resource provision for loT through machine learning in Fog computing," 2019 IEEE Int. Conf. Syst. Comput. Autom. Networking, ICSCAN 2019, Mar. 2019, doi: 10.1109/ICSCAN.2019.8878821.

35. D. Digitalcommons@uri and N. Constant, “An Intelligent Fog Centric Network for IoT-Driven Smart Communities," Open Access Master's Theses, Jan. 2017, doi: 10.23860/thesis-constant-nicholas-2017.

36. F. F. Borelli, G. Biondi, F. Horita, and C. Kamienski, "Architectural Software Patterns for the Development of IoT Smart Applications," Mar. 2020, Accessed: Nov. 11, 2021. [Online]. Available: https://arxiv.org/ abs/2003.04781v2.

37. L. A. Steffenel, M. Kirsch Pinheiro, and C. Souveyet, "Assessing the impact of unbalanced resources and communications in edge computing," Pervasive Mob. Comput., vol. 71, p. 101321, Feb. 2021, doi: 10.1016/J.PMCJ.2020.101321.

38. R. Mahmud, S. N. Srirama, K. Ramamohanarao, and R. Buyya, "Quality of Experience (QoE)-aware placement of applications in Fog computing environments," J. Parallel Distrib. Comput., vol. 132, pp. 190-203, Oct. 2019, doi: 10.1016/J.JPDC.2018.03.004. 
39. J. Mocnej et al., "Quality-enabled decentralized IoT architecture with efficient resources utilization," Robot. Comput. Integr. Manuf., vol. 67, p. 102001, Feb. 2021, doi: 10.1016/J.RCIM.2020.102001.

40. G. Carvalho, B. Cabral, v. Pereira, and J. Bernardino, "Computation offloading in Edge Computing environments using Artificial Intelligence techniques," Eng. Appl. Artif. Intell., vol. 95, p. 103840, Oct. 2020, doi: 10.1016/J.ENGAPPAI.2020.103840.

41. J. Queiroz, P. Leitão, J. Barbosa, and E. Oliveira, "Distributing intelligence among cloud, fog and edge in industrial cyber-physical systems," 16th Int. Conf. Informatics Control. Autom. Robot. ICINCO 2019, vol. 1, pp. 447-454, 2019, doi: 10.5220/0007979404470454.

42. E. Peltonen, T. Leppänen, and L. Lovén, “EdgeAl: Edge-native Distributed Platform for Artificial Intelligence," 2019.

43. M. García-Valls, A. Dubey, and V. Botti, "Introducing the new paradigm of Social Dispersed Computing: Applications, Technologies and Challenges," J. Syst. Archit., vol. 91, pp. 83-102, Nov. 2018, doi: 10.1016/J. SYSARC.2018.05.007.

44. H. Bangui, S. Rakrak, S. Raghay, and B. Buhnova, "Moving to the Edge-Cloud-of-Things: Recent Advances and Future Research Directions," Electron. 2018, Vol. 7, Page 309, vol. 7, no. 11, p. 309, Nov. 2018, doi: 10.3390/ELECTRONICS7110309.

45. J. Edinger, “Context-aware task scheduling in distributed computing systems," 2019, Accessed: Nov. 11, 2021. [Online]. Available: https://madoc.bib.uni-mannheim.de/51051.

46. D. Schäfer, "Elastic computation placement in edge-based environments," 2019, Accessed: Apr. 17, 2021. [Online]. Available: https://madoc.bib.uni-mannheim.de/48832.

47. R. B. Almeida et al., "A distributed event-driven architectural model based on situational awareness applied on internet of things," Inf. Softw. Technol., vol. 111, pp. 144-158, Jul. 2019, doi: 10.1016/j. infsof.2019.04.001.

48. C. Mechalikh, H. Taktak, and F. Moussa, "A Scalable and Adaptive Tasks Orchestration Platform for IOT," in 2019 15th International Wireless Communications Mobile Computing Conference (IWCMC), 2019, pp. 1557-1563, doi: 10.1109/IWCMC.2019.8766744.

49. T. Zhang, Z. Shen, J. Jin, X. Zheng, A. Tagami, and X. Cao, "Achieving Democracy in Edge Intelligence: A Fog-Based Collaborative Learning Scheme," IEEE Internet Things J., vol. 8, no. 4, pp. 2751-2761, Feb. 2021, doi: 10.1109/JIOT.2020.3020911.

50. W. Yanez, R. Mahmud, R. Bahsoon, Y. Zhang, and R. Buyya, "Data Allocation Mechanism for Internetof-Things Systems With Blockchain," IEEE Internet Things J., vol. 7, no. 4, pp. 3509-3522, Apr. 2020, doi: 10.1109/JIOT.2020.2972776.

51. R. I. Ciobanu, C. Negru, F. Pop, C. Dobre, C. X. Mavromoustakis, and G. Mastorakis, "Drop computing: Ad-hoc dynamic collaborative computing," Futur. Gener. Comput. Syst., vol. 92, pp. 889-899, Mar. 2019, doi: 10.1016/j.future.2017.11.044.

52. T. Leppänen and J. Riekki, "Energy Efficient Opportunistic Edge Computing for the Internet of Things," Web Intell. Agent Syst., vol. 17, 2018, doi: 10.3233/WEB-190414.

53. R. Calegari, G. Ciatto, E. Denti, and A. Omicini, “Engineering Micro-intelligence at the Edge of CPCS: Design Guidelines," 2019, pp. 260-270.

54. L. U. Khan et al., "Federated Learning for Edge Networks: Resource Optimization and Incentive Mechanism," IEEE Commun. Mag., vol. 58, no. 10, pp. 88-93, Oct. 2020, doi: 10.1109/MCOM.001.1900649.

55. Z. Lv, D. Chen, R. Lou, and Q. Wang, "Intelligent edge computing based on machine learning for smart city," Futur. Gener. Comput. Syst., vol. 115, pp. 90-99, Feb. 2021, doi: 10.1016/j.future.2020.08.037. 
56. B. D. Deebak, F. Al-Turjman, M. Aloqaily, and O. Alfandi, “IoT-BSFCAN: A smart context-aware system in IoT-Cloud using mobile-fogging," Futur. Gener. Comput. Syst., vol. 109, pp. 368-381, Aug. 2020, doi: 10.1016/j.future.2020.03.050.

57. S. K. Sharma and X. Wang, "Live Data Analytics with Collaborative Edge and Cloud Processing in Wireless IoT Networks," IEEE Access, vol. 5, pp. 4621-4635, 2017, doi: 10.1109/ACCESS.2017.2682640.

58. J. Al-Jaroodi and N. Mohamed, "PSCPS: A distributed platform for cloud and fog integrated smart cyberphysical systems," IEEE Access, vol. 6, pp. 41432-41449, Jul. 2018, doi: 10.1109/ACCESS.2018.2856509.

59. D. Happ and A. Wolisz, "Towards gateway to Cloud offloading in IoT publish/subscribe systems," in 2017 Second International Conference on Fog and Mobile Edge Computing (FMEC), May 2017, pp. 101106, doi: 10.1109/FMEC.2017.7946415.

60. J. An et al., "EiF: Toward an Elastic IoT Fog Framework for Al Services," IEEE Commun. Mag., vol. 57, no. 5, pp. 28-33, May 2019, doi: 10.1109/MCOM.2019.1800215.

61. A. Malekian Borujeni, M. Fathy, and N. Mozayani, “A hierarchical, scalable architecture for a real-time monitoring system for an electrocardiography, using context-aware computing," J. Biomed. Inform., vol. 96, p. 103251, Aug. 2019, doi: 10.1016/J.JBI.2019.103251.

62. P. Y. Zhang, M. C. Zhou, and G. Fortino, "Security and trust issues in Fog computing: A survey," Futur. Gener. Comput. Syst., vol. 88, pp. 16-27, Nov. 2018, doi: 10.1016/J.FUTURE.2018.05.008. 